\title{
Carbonation of Reinforced Concrete Structures
}

\author{
Malek Jedidi*1,2 and Abdessalem Belhassen ${ }^{1}$ \\ ${ }^{1}$ Higher Institute of Technological Studies of Sfax, Department of Civil Engineering, Sfax, Tunisia \\ ${ }^{2}$ University of Tunis El Manar, National Engineering School of Tunis, Civil Engineering Laboratory, Tunis, Tunisia
}

*Corresponding author: Malek Jedidi, Higher Institute of Technological Studies of Sfax, Department of Civil Engineering, Sfax, Tunisia.

Received Date: February 15, 2020

Published Date: March 03, 2020

\begin{abstract}
This paper presents a general overview of the phenomenon of carbonation of concrete which is inseparable from this building material. Indeed, during the lifetime of the structure, carbon dioxide in the atmosphere enters the concrete from the surface of the material. $\mathrm{Carbon}$ dioxide $\left(\mathrm{CO}_{2}\right)$ can then react with the products resulting from the hydration of the cement. Carbonation gradually changes the chemical composition and internal microstructure of concrete. During the life of the structure, the concrete thus traps carbon dioxide up to 10 to $15 \%$ of the $\mathrm{CO}_{2}$ emitted during the decarbonation of the limestone necessary for the manufacture of the cement. Engineers learned during the 20th century to master certain problematic consequences of the phenomenon of carbonation. At the end of the life of the structure, carbonation can, this time, be exploited to fix $\mathrm{CO}_{2}$ in the hardened cement paste of demolition concrete. Recent results show that it is then possible to recapture up to 50 to $60 \%$ of additional $\mathrm{CO}_{2}$. Furthermore, this carbonation is particularly favorable for improving the quality of the recycled aggregate from demolition concrete, thereby facilitating its reuse.
\end{abstract}

Keywords: Carbonation; Concrete; Carbon dioxide; Carbonation depth

\section{Introduction}

Man is neither the first nor the only builder on our planet to use calcium as an essential element in his constructions. Molluscs or planktonic algae thus also secrete mainly their shells based on calcium carbonate or limestone. These two constructive practices have in common that they are part of the calcium cycle on the surface of our planet. This cycle is of paramount importance in current environmental issues since, first of all, limestone is the dominant component of our earth's crust and that the calcium cycle and the $\mathrm{CO}_{2}$ cycle are, as we will see, closely linked. Where molluscs take calcium in solution from their environment and carbonate using atmospheric $\mathrm{CO}_{2}$ to then die and deposit their shells which will then constitute a source of calcium that can be slowly dissolved, the man, more pressed, extracted from limestone from our earth's crust to make cementitious materials. The manufacture of Portland clinker, the main component of modern cements since the end of the $19^{\text {th }}$ century, thus requires the decarbonation of limestone, the most stable form of calcium in nature, and its cooking in the presence of silica. The hydration reaction of clinker in the presence of water leads | to the formation of mineral compounds (hydrates) at the origin of the resistance of concrete or mortar. These chemical compounds are said to be metastable. This means that they are not chemically stable but that their rate of transformation is so slow that they exhibit the appearance of stability on sufficiently short observation time scales. The mere presence of atmospheric $\mathrm{CO}_{2}$, however, leads to the irreversible carbonation of these hydrates in the form of limestone and silica gel, thus completing the mineral calcium cycle on a more or less long-time scale. The carbonation of concrete is therefore an unavoidable phenomenon participating in the calcium cycle on the surface of our planet. The real question, in this context, is not whether a cementitious material will carbonate or not, but on what time scale it will do so and what the consequences will be. It is then possible to distinguish two fundamental time scales. The firsttime scale concerns the lifetime of the cementitious material in the constructed work or the building in service. This timescale is in the order of a hundred years. During this time, the material carbonates under the effect, in general, of atmospheric $\mathrm{CO}_{2}$. The consequences 
of this carbonation, as we will see below, are varied and their predictions are complex.

In this phase, the engineers are particularly attentive to the chemical modifications linked to the carbonation of the composition of the water located in the porosity of the material. If these modifications exceed certain limits, corrosion of the reinforcements in the case of reinforced concrete or the development of microorganisms in the case of a facade plaster can be favored. The second time scale concerns the fate of the material when it is recycled or stored. This phase starts at the end of the life of the structure or building. Usually, this phase starts on average a hundred years after the construction phase, but this duration can be extremely variable. Carbonation can, this time, be used to fix $\mathrm{CO}_{2}$ in the cement matrix at the end of the product's life. In terms of carbonation kinetics, the particularity of the demolition concrete crushing process makes it possible to multiply by a factor close to 1,000 the surface offered to atmospheric $\mathrm{CO}_{2}$ during the transformation of an element into reinforced concrete of the veil or slab type within a structure whose typical thickness is of the order of $20 \mathrm{~cm}$ in centimeter- sized ag- gregates. Recent results show that it is then possible, in the case of traditional concrete from structures from the 50 s and 80 s, to recapture up to 50 to $60 \%$ of the $\mathrm{CO}_{2}$ emitted during the decarbonation of limestone. This carbonation is particularly favorable to an improvement in the quality of the recycled aggregate obtained from demolition concrete.

\section{The Mechanisms of Carbonation}

The atmosphere of our planet contains on average 0.03 to $0.04 \%$ of $\mathrm{CO}_{2}$. This concentration of carbon dioxide varies locally with temperature, atmospheric pressure and the possible proximity of an agglomeration. In highly industrialized areas, in car parks and around major highways, the amount of atmospheric $\mathrm{CO}_{2}$ can be multiplied by a factor of three or four to reach $0.1 \%$.

\section{$\mathrm{CO}_{2}$ at the origin of carbonation}

$\mathrm{CO} 2$ in the atmosphere can cause carbonation of cement matrices. It then diffuses in gaseous form into the porosity of concrete or mortar and dissolves, forming acids on contact with the interstitial solution contained in the cement paste (Figure 1).
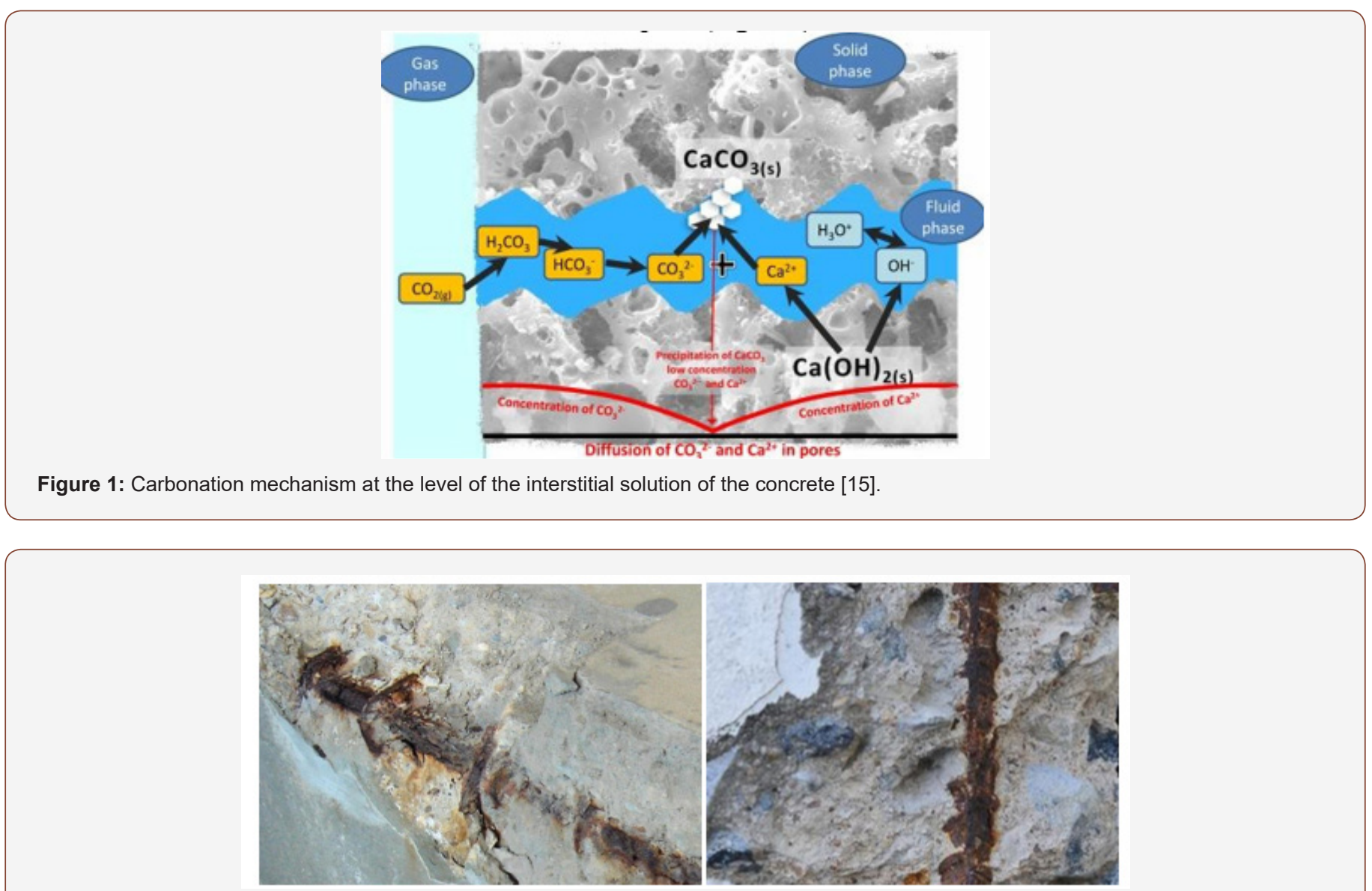

Figure 2: Corrosion of reinforcement due to carbonation of concrete.

This phenomenon has the effect of lowering the $\mathrm{pH}$ of the medium and leading to the dissolution of the hydrates of the cement. Calcium hydroxide $\left(\mathrm{Ca}(\mathrm{OH})_{2}\right)$ is particularly affected [1-3]. More commonly called portlandite, this hydrate plays a fundamental role in maintaining a high $\mathrm{pH}$ protecting the reinforcements of reinforced concrete or preventing the formation of microorganisms. When the portlandite has been completely consumed, or when it is no longer sufficiently accessible to regulate the $\mathrm{pH}$ by buffering 
effect, the medium becomes acidic, thus allowing corrosion of the reinforcements (Figure 2).

Carbonation of hydrated calcium silicates (C-S-H) is also possible. The reaction is represented chemically as follows:

$$
\mathrm{CO}_{2}+\mathrm{Ca}(\mathrm{OH})_{2} \Leftrightarrow \mathrm{CaCO}_{3}+\mathrm{H}_{2} \mathrm{O}
$$

The C-S-H carbonate according to a progressive mechanism of decalcification, the calcium ions coming to precipitate in the porosity with the carbonate ions in the form of calcium carbonate. Decalcification leads to the formation of a very amorphous (highly polymerized) and more or less hydrated silica gel. The carbonation of portlandite and C-S-H is inevitable, even for very low $\mathrm{CO}_{2}$ concentrations $(0.03 \%)$. The $\mathrm{CO}_{2}$ concentration simply comes to control the speed at which this phenomenon will take place and the speed with which it will penetrate into the material (this is called penetration kinetics).

\section{Carbonation changes the $\mathrm{pH}$ of concrete}

Note that cements also contain alkali metal oxides $\left(\mathrm{Na}_{2} \mathrm{O}\right.$ and $\mathrm{K}_{2} \mathrm{O}$ ) which are found in the form of alkaline bases in the interstitial solution $(\mathrm{NaOH}$ and $\mathrm{KOH})$. These alkaline species make it possible to maintain a basicity beyond the value imposed by the portlandite (that is to say beyond 12.4 at $20^{\circ} \mathrm{C}$ ). However, their carbonation is very rapid since the alkalis are almost only present in aqueous form and are therefore easily leached (we also say leached) in the presence of rain. Cracking of cementitious materials (under the effect of loads and / or tensile stresses generated by thermal shrinkage, self-drying and drying) facilitates the penetration of $\mathrm{CO}_{2}$ and can reduce the service life of reinforced structures [4,5]. During the carbonation process, the main element of transformation is calcium carbonate $\left(\mathrm{CaCO}_{3}\right)$ of which there are several polymorphic varieties: calcite, vaterite and aragonite. Calcite is the most stable variety and corresponds to the form of limestone which was used to obtain the clinker after cooking the raw in the cement kiln. From the point of view of reinforced or prestressed concrete structures, carbonation is a deleterious phenomenon which progresses from the outside (facing) towards the inside, through the area of coating of the reinforcements, according to a physicochemical process which associates diffusion of $\mathrm{CO}_{2}$ through porosity and chemical reactions of $\mathrm{CO}_{2}$ fixation in hydrates (Figure 3).

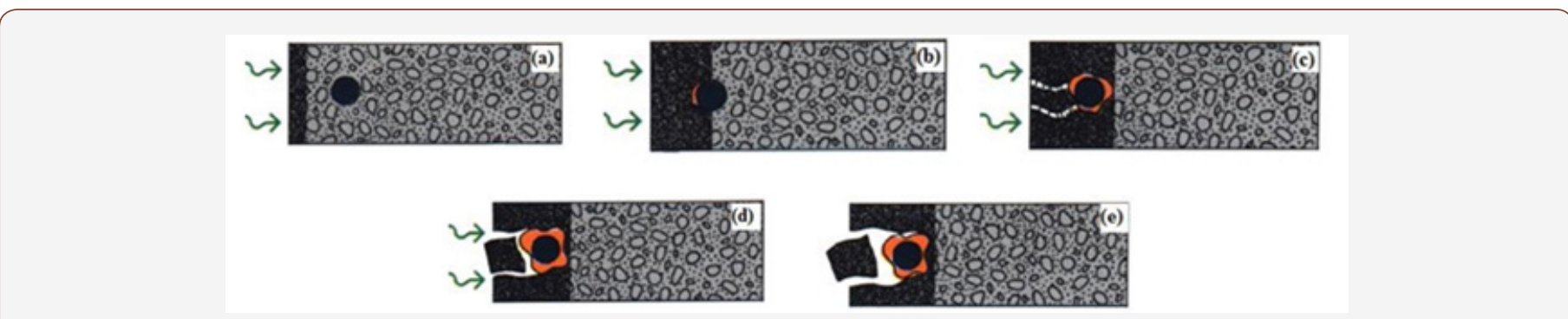

Figure 3: Carbonation process. $(A)$ : Alkaline concrete $(\mathrm{pH}=12.5)$; $(\mathrm{B}): \mathrm{CO}_{2}$ penetration = Carbonation, alkalinity lower than $\mathrm{pH}=9$; $(\mathrm{C})$ : Penetration of water and oxygen leads to oxidation of the metal reinforcement; (D): Rusting causes cracking of the concrete; (E): Expansion of rust causes the concrete to burst.

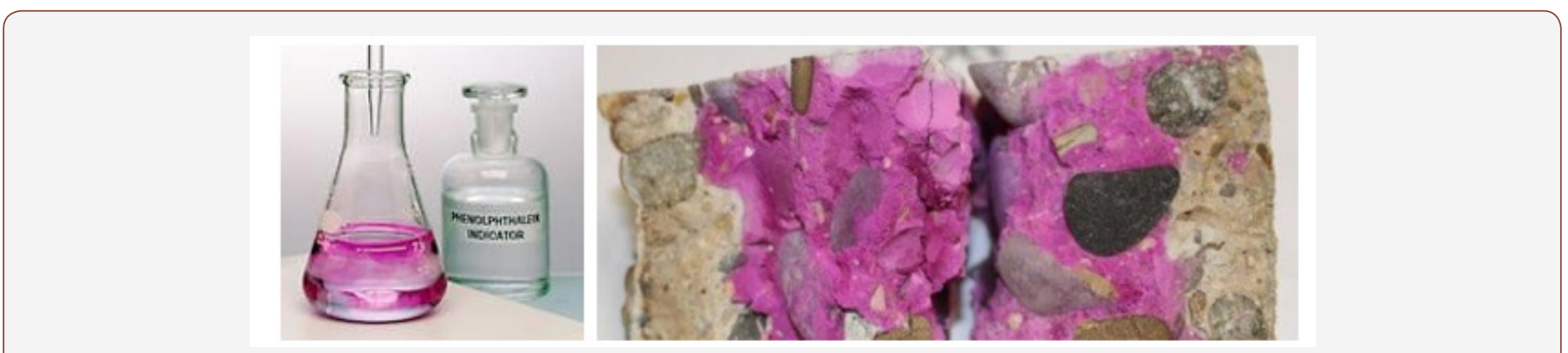

Figure 4: Carbonation of concrete (non-colored area) Phenolphthalein test.

Neutralizing the alkalinity of the resulting medium becomes problematic when it reaches the first bed of the reinforcements. Indeed, if the $\mathrm{pH}$ decreases too much, the passivation layer of steels made up of ferrous oxides and hydroxides becomes unstable. This results in rapid corrosion, known as generalized, over the entire surface of the reinforcement. Corrosion products, which are expansive, damage the facing (cracking, spalling, etc.), form unsightly rust flows and lead to a reduction in the section of steel in the rein- forcements. The threshold $\mathrm{pH}$ value below which carbonation can lead to marked corrosion of reinforcements is still debated in the literature. Located between 9 and 11, this threshold depends on the nature of the cement and especially on the hydric state of the concrete; in particular, enough liquid water is required to facilitate ion exchange at the level of the micropiles which appear on the surface of the steel, without however blocking the diffusion of corrosive oxygen in the gas phase. It is possible to carry out a diagnosis of 
the carbonation state of the concrete by removing from a facing a core which is split in two and on which a colorimetric $\mathrm{pH}$ indicator (phenolphthalein) is sprayed. The carbonate part appears colorless and the non-carbonate nucleus, that is to say very basic, is colored pink (Figure 4).

However, it is stipulated in European regulations that phenolphthalein has a carcinogenic character. Its use should be limited or severely controlled. This is why the scientific community is looking into the use of a new colored $\mathrm{pH}$ indicator. The colorimetric method does not provide information on the true shape of the carbonation front as illustrated in Figure 5 where the reinforcements appear protected, because being located downstream of the front detected by projection of phenolphthalein, when in reality the level carbonation would be sufficient to initiate corrosion. To gain precision, there are more expensive methods such as thermogravimetric analysis (performed on a succession of thin slices of material taken by sawing from the surface) and gamma-densimetry (non-destructive method). The use of several colored indicators presenting different $\mathrm{pH}$ shift zones could also be a solution provided that the color contrasts between the healthy and carbonate zones are sufficiently marked. Air humidity is a very important factor since carbonation is slow, or even absent, in an atmosphere saturated with water (diffusion of $\mathrm{CO}_{2}$ in the gas phase is then almost impossible if the porosity of the concrete is saturated) and in an atmosphere that is too dry (the dissolution of $\mathrm{CO}_{2}$ in the aqueous phase does not take place because it is kinetically very limited). This behavior leads to optimal carbonation for a relative humidity between 50 and $70 \%$ $[6,7]$. On the other hand, note that corrosion will be optimal for a relative humidity of around $90 \%-95 \%$. From a normative point of view, the risk of carbonation is taken into account to formulate a concrete (W/C ratio, cement dosage and mineral additions content) through the BS EN 206-1standard. It is interesting to note that the most damaging exposure class with respect to carbonation $\left(\mathrm{XC}_{4}\right)$ corresponds to a climate with alternating dry and wet phases. This situation is certainly not the most favorable from the point of view of carbonation alone; however, given the risk of corrosion, it maximizes the kinetics. Note also the existence of standards (Eurocode 2) allowing the dimensioning of the coating thickness according to the concrete resistance class and the aggressiveness of the environment vis-à-vis the attack of $\mathrm{CO}_{2}$.

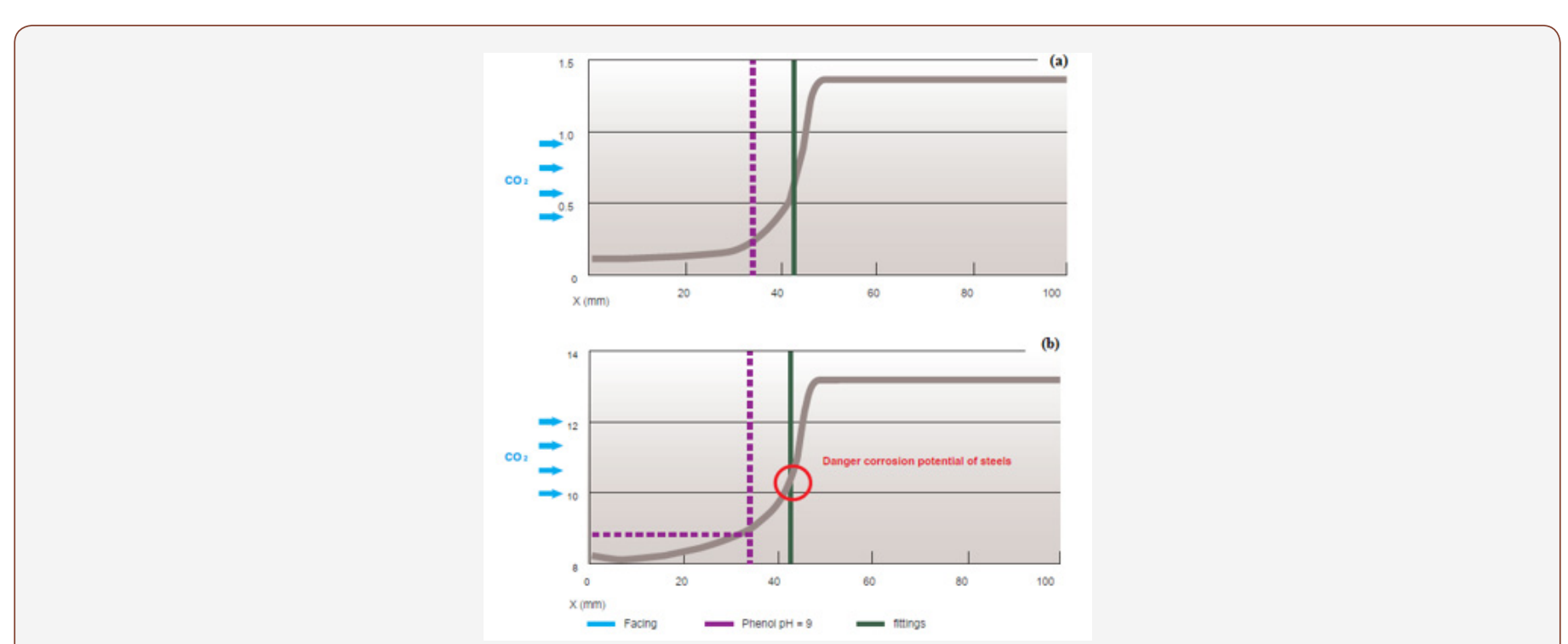

Figure 5: Progression of carbonation. (A): Diagnosis of carbonation state of a concrete with phenolphthalein spray. (B): Mapping of the carbonation front and its position relative to reinforcement.

\section{Parameters influencing carbonation kinetics}

There are many carbonation models which translate, more or less directly, the physico- chemical resistance of concrete against the penetration of the carbonation front. The parameters used relate to:

1. The porosity of the concrete which conditions the "physical" resistance of the material to the diffusion of $\mathrm{CO}_{2}$.

2. The carbonate hydrate content: the higher it is, the more the concrete is able to "chemically" slow down the penetration of the carbonation front.
3. The microstructure of the material (pore size) on which the water state of the material depends which will condition the kinetics of penetration of the phenomenon. It is therefore essentially the material's permeability to liquid water that will be a relevant indicator of durability.

These models generally lead to a slow evolution in square root of time from the depth of carbonation. To fix ideas, with ordinary concrete kept in an environment whose relative humidity is between 50 and $70 \%$, the depth of carbonation is $5 \mathrm{~mm}$ after one year, $10 \mathrm{~mm}$ after 4 years and $25 \mathrm{~mm}$ after 25 years. The approximate depth of carbonation can be calculated using the following formula [8]: 


$$
D=K .(t)^{\lambda}(2)
$$

Where, $\mathrm{D}$ is the carbonation depth ( $\mathrm{mm}), \mathrm{KC}$ is the carbonation coefficient, a function of the quality of the concrete and its exposure $[\mathrm{mm} /$ (years) $1 / 2]$, $\mathrm{t}$ is the age of concrete (years), $\lambda$ is generally taken equal to 0.5 . Numerous investigations attest that the humidification-drying cycles tend to reduce the rate of carbonation, by lowering the parameter $\lambda$ to values close to $0.25-0.35[9,10]$. The models are used to predict the lifetime of the concrete material against the initiation of carbonation corrosion. They therefore often have the defect of not taking into account the kinetics of corrosion and, consequently, they lead to an oversizing of the coating thickness and to an often too safe formulation of the concrete. This approach to concrete design is increasingly questioned in the current context of preserving non-renewable resources and limiting the carbon footprint of concrete construction. The mineral additions commonly used consume part of the portlandite by means of pozzolanic reactions (case of fly ash), while giving the material reduced porosity and good mechanical properties thanks to the fineness of the particles used [11-14]. Carbonation can also be the cause of disorders in façade coatings, generally corresponding to mortars with a high proportion of partially carbonated lime $\left(\mathrm{Ca}(\mathrm{OH})_{2}\right)$. The acidification of the medium which results from the attack of $\mathrm{CO}_{2}$ can be at the origin of the development of microorganisms (lichens, mosses, algae, etc.) which do not grow in a strongly basic medium. These microorganisms are responsible for unsightly soiling. It is possible to temporarily remove these traces by washing the wall with diluted bleach or using a suitable fungicide product. When the coating dries quickly enough, the water deposits the lime salts inside the fine channels which are the pores of the coating. Carbonation is then invisible. But when the coating dries slowly (humid air, temperature below $8-10^{\circ} \mathrm{C}$ ), the white deposit forms on the exterior surface of the coating [15].

\section{The Benefits of Carbonation for Concrete}

Unlike the harmful consequences of carbonation on the durability of concrete structures, carbonation is rather a beneficial phenomenon from the point of view of the concrete material itself. It is indeed recognized that the carbonation of cement matrices based on CEM I type cement is achieved with a mass gain corresponding to the fixation of $\mathrm{CO}_{2}$ in the hydrates. The fact that the molar volume of the calcium carbonate formed is greater than that of the hydrates leads to clogging of the microstructure which makes the concrete less permeable to aggressive agents (in this case $\mathrm{CO}_{2}$ and $\mathrm{O}_{2}$ gases, but also sulphate ions, chlorides, and more generally, aggressive waters such as seawater, selenite or magnesian waters). It has even been shown that carbonation makes it possible to increase the mechanical resistance of concrete and to make the material more chemically stable vis-à-vis swelling reactions (internal or external sulfatic reaction, alkali-aggregate reaction). On the other hand, the modulus of rigidity or modulus of elasticity is increased, which is not always perceived as an advantage because this can lead to embrittlement of thin parts and to a heterogeneity of resistance and shrinkage in the case of coatings of partially carbonated facades where surface cracks can be observed which stop at the level of the non-carbonate internal zone.

This improvement in the properties of concrete has even been used in some countries for the prefabrication of blocks by autoclaving using high concentrations of $\mathrm{CO}_{2}$. We also know that the ancients (Etruscans, Phoenicians, Greeks, Romans, and even Chinese) used atmospheric carbonation to make masonry joints and plasters based on fatty lime mortar. The hardening took place by carbonation under the action of $\mathrm{CO}_{2}$ from the atmosphere. This fatty lime could not be taken in water, but only in air, hence its name "air lime" and the qualification of "air intake" in this case. The hardening was rapid on the surface, then the rate of carbonation slowed down by the dense layer of calcium carbonate formed. This crust was impermeable such as can still be found at the heart of the thick old walls of oily lime, still plastic and not carbonated, behind a compact layer of calcite. Currently, there is an upsurge in the use of mortars and aerial paints in the building sector. In terms of pore size distribution in the cement matrix, carbonation leads to a rearrangement of the porous distribution with the creation of macropores (mainly for matrices with high W/C ratio exposed to high CO2 contents), and this despite an overall reduction in porosity. This phenomenon is illustrated in Figure 6 in the case of building concrete with high $\mathrm{W} / \mathrm{C}=0.84$ (Figure 6).

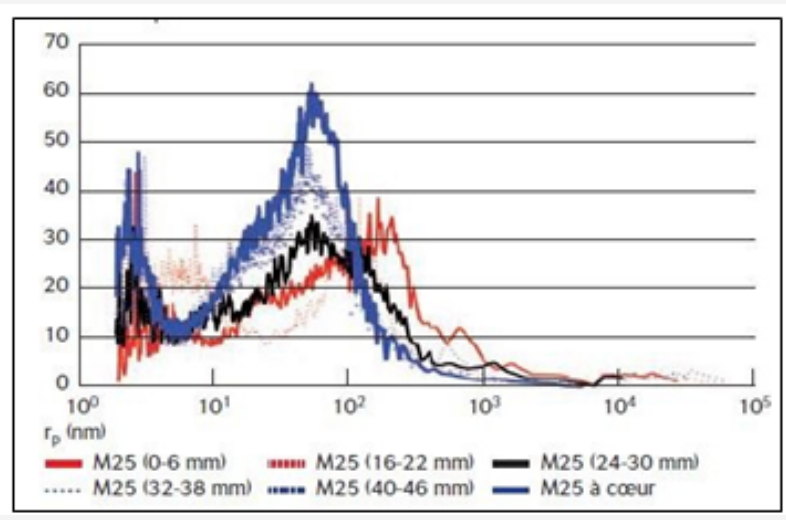

Figure 6: Distribution of pore size obtained by porosimetry by mercury intrusion on a building concrete of E/C $=0.84$ from the carbonate surface towards the healthy heart $\left(50 \% \mathrm{CO}_{2}\right)$. 
The positive aspects of carbonation which have just been described are essentially characteristic of cement systems containing a high dosage of CEM I type cement. As soon as a significant proportion of blast furnace slag, fly ash or smoke silica is added, the properties of the material deteriorate as a result of carbonation: drop in density, reduction in resistance, significant opening of porosity, etc. It would seem that this peculiarity is to be correlated with the fact that hydrates of the C-S-H type formed from the additions lose, during carbonation, a significant amount of water of chemically bound structure. The carbonation is responsible for whitish efflorescence of calcium carbonate on the surface of the facings, without any inconvenience, except from an aesthetic point of view. This phenomenon occurs if the portlandite is dissolved by the impregnation water during the humid climatic phases and the calcium ions migrate towards the surface by capillarity during the drying phases. Driven out, the calcium ions carbonate in the air. Streaks of calcite are then seen on the walls of the structures in the vicinity of the cracks and fill the most porous areas. One can notice in the dam galleries the formation of calcite stalactites coming from the washing of the portlandite.

\section{Conclusion}

The carbonation of concrete can be an evil or a good, depending on the design and destination of the works. Good knowledge of this phenomenon makes it possible to take advantage of this property, either by protecting the concrete from this carbonation (suitable painting for example), or by limiting it (increasing the coating thickness of the reinforced concrete, improving the quality of the coating concrete), or on the contrary by promoting it (thanks to accelerated carbonation). Taking advantage of carbonation is especially useful to improve the properties of unarmored cement systems, but also to trap $\mathrm{CO}_{2}$ which is an environmental aspect far from being negligible. It is now possible, by following the prescriptions of the standards, to control the effects of the carbonation of concrete on the reinforcements during the period of use of the structure by adjusting the formulation of the concretes and by optimizing the value of the coating of the reinforcements depending on the various attacks and aggressions (translated by the notion of exposure class) that concrete will undergo. In addition, engineers currently have at their disposal performance tools based on the evaluation of sustainability indicators (porosity, permeability, portlandite content, ...) and predictive models which allow dimensioning.

\section{Acknowledgement}

None.

\section{Conflict of Interest}

No conflict of interest.

\section{References}

1. Houst YF, Wittmann FH (1994) Influence of porosity and water content on the diffusivity of $\mathrm{CO} 2$ and 02 through hydrated cement paste. Cement and Concrete Research 24(6): 1165-1176.

2. Ngala VT, Page CL (1997) Effects of carbonation on pore structure and diffusional properties of hydrated cement pastes. Cement and Concrete Research 27(7): 995-1007.

3. Thiery M, Villain G, Platret G (2003) Effect of carbonation on density, microstructure and liquid water saturation of concrete. Proc. 9th Engineering Conference on Advances in Cement and Concrete, USA, pp. 481-490.

4. Castel A, François R, Arliguie G (1999) Effect of loading on carbonation penetration in reinforced concrete elements. Cement and Concrete Research 29(4): 561-565.

5. Steffens A, Dinkler D, Ahrens H (2004) Modeling carbonation for corrosion risk prediction of concretes structures. Cement and Concrete Research 32(6): 935-941.

6. Saetta AV, Vitaliani RV (2004) Experimental investigation and numerical modeling of carbonation process in reinforced concrete structures - Part I: theoretical formulation. Cement and Concrete Research 34(4): 571579.

7. Tuutti K (1982) Corrosion of steel in concrete, Swedish Cement and Concrete Institute, Stockholm, Europe.

8. Parrott LJ (1987) A review of carbonation in reinforced concrete, Cement and Concrete Association p.42.

9. Daimon M, Akiba T, Kondor R (1971) Through pore size distribution and kinetics of the carbonation reaction of Portland cement mortars. Journal of the American Ceramic Society 54(9): 423-428.

10. Alexander MG, Mackechnie JR, Yam W (2007) Carbonation of concrete bridge structures in three south african localities. Cement and Concrete Composites 29(10): 750-759.

11. Tsukayama R (1980) Long Term Experiments on the Neutralization of Concrete Mixed with Fly Ash and the Corrosion of Reinforcement, Proc. of 7 th International Congressional on the Chemistry of Cement (ICCC), France (3): 30-35.

12. Paillere AM, Raverdy M, Grimaldi G (1986) Carbonation of concrete with low calcium fl y ash and granulated blast furnace slag : influence of air entraining agents and freezing and thawing cycles, Proc. of $2^{\text {nd }}$ International Conference on Fly Ash, Silica Fume, Slag and Natural Pouzzolans in Concrete, Espagne (Madrid), Spain pp.541-562.

13. Roper H, Kirby G, Baweja D (1986) Long term durability of blended cement concretes in structures, Proc. of $2^{\text {nd }}$ International Conference on Fly Ash, Silica Fume, Slag and Natural Pouzzolans in Concrete, Espagne (Madrid), Spain pp.463-482.

14. Skjolsvold O (1986) Carbonation depths of concrete with and without condensed silica fume, Proc. of $2^{\text {nd }}$ International Conference on Fly Ash, Silica Fume, Slag and Natural Pouzzolans in Concrete, Espagne (Madrid), Spain pp.1031-1048.

15. Czarnecki L, Woyciechowski P (2013) Modelling of concrete carbonation; is it a process unlimited in time and restricted in space. Bulletin of the polish academy of sciences technical sciences 61(1): 173-181. 